\title{
Determinación de descriptores sensoriales para un producto untable a base de frijol gandul (Cajanus cajan) mediante dos mini grupos focales
}

\author{
Carmén Andrés Jiménez \\ Universidad Estatal a Distancia (UNED), Apdo 474-2050 Mercedes de Montes de Oca, San José, Costa Rica; candres@uned.ac.cr
}

Recibido 1-XI-2012 Corregido 26-II-2013 Aceptado 15-V-2013

\begin{abstract}
Determination of sensorial attributes of a spread made from pigeon pea (Cajanus cajan) using two mini focus groups. The focus group technique is wide used to determine attributes for an innovating product. The pigeon pea is an alternative crop that offers an option for food security, fixes nitrogen in the soil, protects river basins and has a high nutritional value. Two mini focus groups were used to identify the most important sensorial descriptors of a hummus-like spreadable product made from the folk variety of pigeon pea. The techniques that were used were: drawing, planetarium, sampling and comparison with competitors. The descriptors obtained from the exercise were: beige color, hummus like taste, small lumps or totally homogeneous, oxibiodegradable packaging, transparent lid, no colorants or preservatives and spreadable texture. It was categorized as a natural, healthy and convenience product that is preferred to be accompanied with pita bread and chips. The jalapeño hummus was preferred, followed by the traditional flavor and finally the dill and celery flavor. I recommend additional sensorial analysis, physical chemical and microbiological tests, a market study and a Quality Function Development before launching the product to the market.
\end{abstract}

\section{KEY WORDS}

Pigeon pea, mini focus group, hummus, attributes, sensorial analysis, consumer requirements

\section{RESUMEN}

La técnica denominada grupo focal se utiliza ampliamente en la determinación de descriptores para un producto innovador. El gandul un cultivo alternativo que contribuye a la seguridad alimentaria, fija nitrógeno en el suelo, protege las cuencas hídricas y de alto valor nutricional. Por medio de dos mini grupos focales se determinaron los descriptores sensoriales más importantes para un producto untable tipo hummus a base de la variedad criolla de gandul. Las técnicas utilizadas fueron: dibujo, planetario, degustación y comparación con la competencia. Los descriptores más sobresalientes fueron color beige, sabor característico a hummus, ajo y picante, apariencia con grumos pequeños o totalmente homogénea, empaque oxibiodegradable, con tapa transparente, sin colorantes o preservantes y textura untable. Se categorizó como producto natural, saludable y de conveniencia que se prefiere acompañar con pan pita y chips. La degustación colocó al hummus de chile jalapeño como el más aceptado, seguido por el tradicional y por último el de perejil y culantro. Se recomienda realizar más análisis sensoriales, análisis físico químicos y microbiológicos, realizar un estudio de mercado basado en estos grupos focales y construir un QFD (Quality Function Development) para lanzar el producto al mercado.

\section{PALABRAS CLAVE}

Gandul, mini grupo focal, hummus, descriptores, análisis sensorial, requerimientos del consumidor
El gandul es una leguminosa originaria de la India, Asia y Africa y se cultiva en diferentes países de Latinoamérica como Puerto Rico, Perú, República Dominicana y Panamá (Ocampo, 2010). Recientemente, gracias al proyecto denominado "Desarrollo de fincas agrosilvopastoriles integrales y productos nutritivos a base de gandul" liderado por un grupo de cinco investigadores de la Escuela de Ciencias Exactas y naturales de la UNED (del cual la autora lidera la parte de desarrollo de productos nuevos), se volvió a incursionar su cultivo en Costa Rica, especialmente en la zona de La Tigra y Chachagua, en San Carlos.

A partir de su cultivo, se han logrado introducir más de ocho variedades en el campo y se ha determinado que por generar mayor volumen en su cosecha y tener mayor resistencia al clima de la zona, las más útiles según Céspedes (comm pers, 2012) son las siguientes: Catie (variedad negra), Criolla (variedad color crema) y Panamá (variedad color crema mezclada con negro). 
El gandul tiene muchas propiedades, entre ellas las nutritivas, ya que sus granos contienen de 18 a $25 \%$ de proteína, oligoelementos y vitaminas A y $C$ (cinco veces más que la arveja) (Liberterre, SF). Debido a lo anterior, se ha utilizado como sustituto de la proteína comercial (gallinaza) para alimentar vacas y los resultados han sido sumamente positivos en el ámbito social, económico y ambiental (Hernández \& Izaguirre, SF).

Aunado a lo anterior, el gandul es fijador de nitrógeno en el suelo (Sharma, 1993), protector de cuencas hídricas (Aroca, Izaguirre \& Zelaya, 2008) y una excelente opción para cooperar con la seguridad alimentaria en Costa Rica, ya que sus propiedades nutricionales hacen que sea un sustituto adecuado en porcentajes de 10 a 30 para otros cereales como el arroz, el sorgo y el maíz tierno (Bressani, Gómez \& Gonzaga, 1986).

Basado en las bondades que tiene el gandul, se determinó dentro del proyecto de investigación del que forma parte este artículo, que una de las maneras de explotarlas es desarrollando productos innovadores, el cual tiene como objetivo dar una mejor calidad de vida a de los productores de la zona, además de introducir en el mercado nacional un producto diferente, que complemente la dieta de los costarricenses. Para lo anterior, una de las herramientas para llevarla a cabo es el análisis cualitativo del consumidor por medio de "mini grupo focal".

Un grupo focal se compone de un número máximo de 12 personas, pero actualmente se están realizando cada vez más los llamados mini grupos focales, sobre todo en Inglaterra, para minimizar costos y dar más fluidez al desarrollo de productos (Barrios \& Costell, 2004).

Según hallazgos del campo de la sicología social, cuando se forma un grupo de personas que no se conocen entre sí, se logra que los participantes se sientan más libres y provean respuestas más sinceras (Barrios \& Costell, 2004).

Las ventajas de la técnica del mini grupo focal son las siguientes: es muy funcional, rápido y económico porque los resultados se pueden utilizar de forma útil para tomar decisiones que se deben tomar con rapidez y es confiable porque las respuestas son usualmente sinceras (Barrios \& Costell, 2004).

Por otro lado, el grupo focal presenta la limitación de que no hay respuestas individuales (como en una encuesta), sino que son de grupo y pueden ser influenciadas; hay un número reducido de participantes, lo cual no necesariamente se puede extrapolar a una población más grande y si existe un moderador que no es adecuado, puede haber un mal uso de la información (Barrios \& Costell, 2004).

A partir de los resultados de un grupo focal, se puede utilizar una técnica denominada QFD (Quality Function
Development), por medio de la cual se elabora un diagrama llamado "casa de la calidad", el cual tiene cinco partes: requisitos del consumidor, matriz de planeamiento, requisitos técnicos, interrelaciones, techo y metas (Alpizar, comm pers, 2012).

El QFD provee un enfoque comprensivo y sistemático que asegura que los productos cumplen o superan las expectativas de los consumidores (Erraach, Sayadi \& ParraLópez, 2011) y refleja estas expectativas de una manera más precisa (Alinezad, Seif \& Esfandiari, 2011).

El producto que se escogió para la realización de este estudio fue el producto untable tipo hummus a base de gandul, a partir de las degustaciones que se han realizado del mismo y su buena aceptación.

El hummus es un producto de origen árabe cuyo significado es "garbanzo" y se cree que fue utilizado por los Egipcios hace más de 7000 años y sembrado en el mediterráneo hace 5000 (Boskou, 2012).

El garbanzo tiene propiedades nutricionales también reconocidas, por su alto nivel de lecitina (más que la soya y el maní), vitaminas $B$ y $C$, ácido fólico y fibras solubles (Chaves, 2010).

Con respecto a su valor nutricional, el hummus de garbanzo es alto en ácidos grasos monosaturados, fibra y en fenoles de importancia biológica (Boskou, 2012). Además, es considerado un plato saludable y recomendado para adolescentes (Khan, Saeed, Hameed, Hussain, Amer \& Haq, 2011) y se ha convertido en una opción los planes de dieta de los Estados Unidos (Boskou, 2012).

El hummus de garbanzo es poco conocido en Costa Rica, pero cuenta con un nicho de mercado específico establecido tanto por parte de empresas internacionales como nacionales. Con respecto a esta situación, Stanton (1990), menciona que el motor principal del desarrollo de productos es la competencia, ya que al analizar este segmento del mercado, se logra ofrecer al consumidor un producto de mejor calidad, precio y que solvente mejor sus necesidades.

Por lo tanto, el hecho de lanzar al mercado un producto untable tipo hummus a base de gandul, tomando en cuenta sus propiedades, el hecho de ser un producto elaborado por productores nacionales y ser totalmente orgánico, lo podría hacer atractivo para el consumidor y competitivo ante los productos ya existentes en el mercado.

Para el desarrollo de este producto a base de gandul, se decidió utilizar el nombre "producto untable", no "hummus", dado que no se elabora a base de garbanzo, por recomendaciones de expertos en propiedad intelectual del Registro nacional (Mena, 2012) y el primer instrumento 
utilizado para realizar la investigación de mercado fue la técnica llamada grupo focal.

El objetivo de este artículo fue determinar los descriptores sensoriales de un producto untable tipo hummus a base de gandul, mediante dos mini grupos focales.

\section{METODOLOGÍA}

\section{Pruebas preliminares}

A partir de una feria de gandul llevada a cabo el 21 de Junio del 2012 por el equipo de investigación de gandul de la Escuela de Ciencias Exactas y Naturales (del que la autora forma parte), se dieron a degustar los siguientes platillos:

- Producto untable tipo hummus de gandul (variedad criolla, tres formulaciones de la autora)

- Producto untable tipo hummus de gandul (variedad Catie, receta de la autora)

- Ensalada de Gandul panameño (Amerling, 2012)

- Guiso de Gandul criollo con cerdo (Saravia, 2012)

- Gandul arreglado (variedad Catie, receta de la autora).

- Producto untable tipo hummus light con atún (variedad criolla, receta de la autora)

\section{Mini grupo focal}

Dos mini grupos focales fueron realizados en las instalaciones de la Escuela de Ciencias Exactas y Naturales de la UNED con el objetivo de generar los descriptores más importantes para el producto untable tipo hummus a base de gandul.

La escogencia de los participantes se realizó de acuerdo a dos criterios: consumidores y no consumidores de hummus.

Se escogieron 12 personas (seis participantes en cada sesión) y la actividad se llevó a cabo en una sala de reuniones pequeña. Se proyectó una presentación para ilustrar el producto, ejemplos de competencia, sabores e ingredientes.

Toda la información dada por los participantes se transcribió textualmente, en sus propias palabras como se observa en el Cuadro1 y la información en el Apéndice 1.

Para conducir la sesión se siguieron los siguientes pasos:

Bienvenida y actividad para romper el hielo: Se dio la bienvenida y el agradecimiento a los participantes por haber colaborado con la actividad y se les preguntó qué les gustaba hacer como una actividad para romper el hielo.
Conocimiento previo del hummus: Se preguntó a los participantes qué conocían del hummus y por qué tenían curiosidad de participar. Seguidamente, se les mostró los ingredientes básicos a partir de los cuales estaba hecho: garbanzo, tahini, jugo de limón, ajo, aceite de oliva y especies.

Elaboración de un dibujo: Se aplicó esta técnica para que los participantes dibujaran el hummus perfecto a base de gandul. Se les explicó que si pudieran pedirle a un ingeniero de alimentos que les fabricara este hummus, cómo se lo pedirían (considerando sabor, color, apariencia, material de empaque, ingredientes y textura).

Planetario: Esta actividad se utilizó para que los participantes ordenaran, en orden de preferencia, seis sabores de hummus: ajo rostizado, chile jalapeño, con tomates secos, con aceituna negra, con perejil y culantro y tradicional.

Preferencia para acompañar el hummus: Seguidamente, se les solicitó mencionar una o varias de las siguientes opciones con las cuales les gustaría acompañar el hummus: pan pita, chips, falafel, wraps de vegetales, sándwiches, pretzels, huevo duro, hamburguesas, hot dogs.

Tendencias en el desarrollo de productos: Las siguientes fueron las opciones en las que se podría incluir el hummus de gandul. Los participantes podían escoger una o más: amigable con el ambiente, natural, saludable, conveniencia, exótico, preferido por precio o variado.

Degustación: Se realizó una degustación de hummus de gandul de diferentes tipos: tradicional, jamón, perejil y culantro y chile jalapeño.

Evaluación de la competencia: Como parte final del grupo focal, se analizaron diferentes marcas, tipos de empaque e ingredientes adicionales que podrían ser utilizados en la elaboración del producto de interés.

\section{Análisis estadístico de los resultados}

Debido a la cantidad pequeña de datos, se realizó una evaluación de las respuestas mediante la prueba estadística no paramétrica Wilcoxon (Mann-Whitney U), para determinar diferencias significativas entre las respuestas de ambos grupos evaluados y así ver si las respuestas podían considerarse como un todo o si se debía presentar el resultado de cada grupo por separado. 


\section{RESULTADOS}

\section{Pruebas preliminares}

Los productos que tuvieron la mayor aceptación fueron: el producto untable hummus de gandul (variedad criolla, tres formulaciones de la autora), el producto untable tipo hummus de gandul (variedad Catie, receta de la autora), la ensalada de gandul panameño y el guiso de gandul criollo con cerdo.

Debido a que el producto untable tipo hummus tuvo comentarios muy positivos por parte de las más de 200 personas que asistieron a la feria, se decidió empezar la investigación para desarrollar un producto innovador.

Para la elaboración de este producto, se escogió la variedad criolla debido a que fue la más gustada en la feria, además de que hace que el producto untable tipo hummus tenga una apariencia similar al hummus de garbanzo.

\section{Mini grupos focales}

Conocimiento previo del hummus: En el grupo 1 la mitad de los participantes no conocían el hummus, mientras que en el grupo 2 todos lo conocían.

En ambos grupos existía la curiosidad de probarlo y aprender a hacerlo, además de coincidir en que el hacer un producto untable tipo hummus a base de gandul era una opción alternativa que tendría un alto valor nutricional.

Descriptores del producto untable tipo hummus a base de gandul mediante la técnica del dibujo: La evaluación de las respuestas de los participantes de ambos grupos mediante la prueba estadística Wilcoxon (Mann-Whitney U), muestra que en ninguno de los casos existen diferencias significativas entre los grupos como para analizar las respuestas de cada uno por separado.

Es por lo anterior que en las figuras de la 1 a la 6 se muestran los promedios de las respuestas del grupo 1 y grupo 2 para cada descriptor del producto untable tipo hummus.

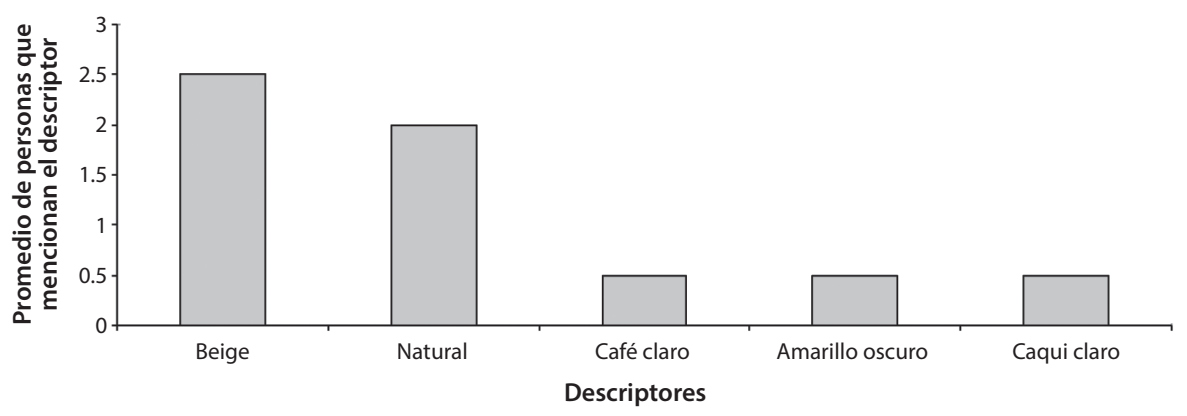

FIG. 1. Descriptores más representativos del color del producto ideal generados en promedio por ambos grupos focales ( $p=0,9134)$.

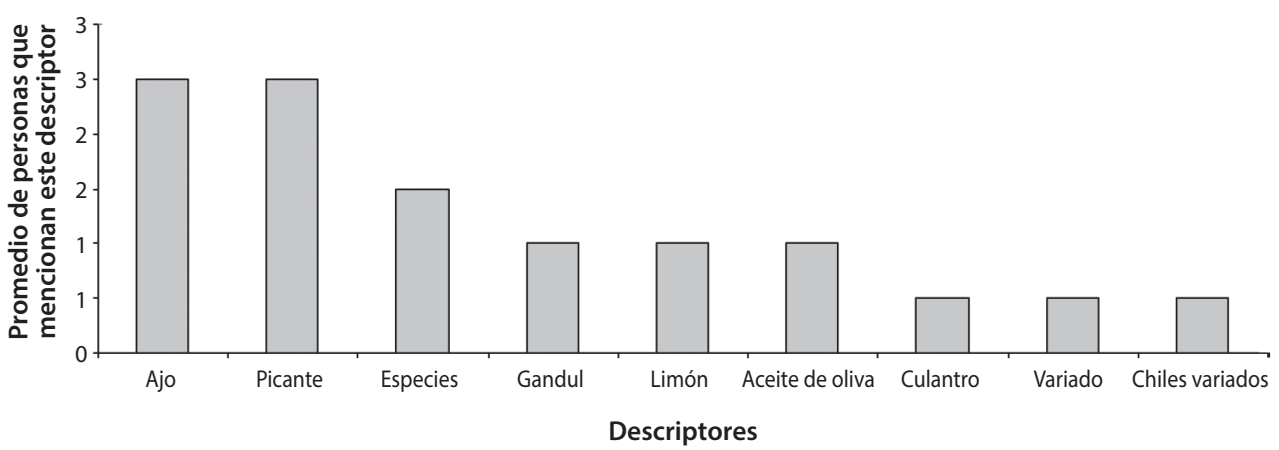

FIG. 2. Descriptores más representativos del sabor del producto ideal generados por ambos grupos focales $(p=0,6355)$. 


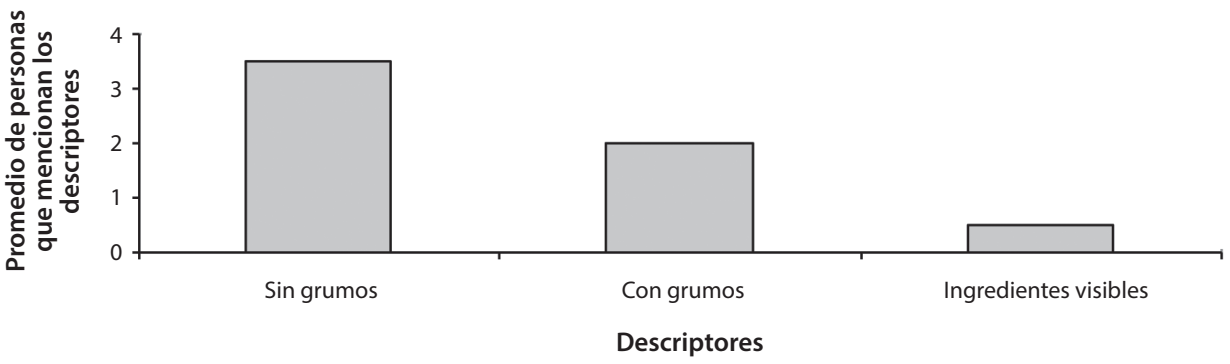

FIG. 3. Descriptores más representativos de la apariencia del producto ideal generados por ambos grupos focales $(p=0,9999)$.

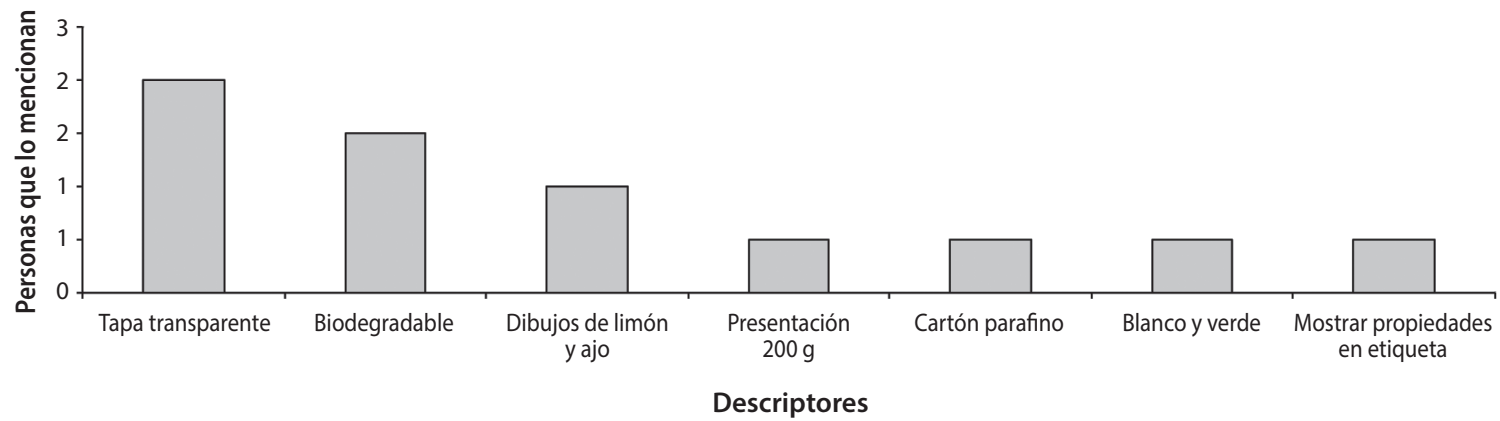

FIG. 4. Descriptores más representativos del empaque del producto ideal generados por ambos grupos focales ( $p=0,3957)$.

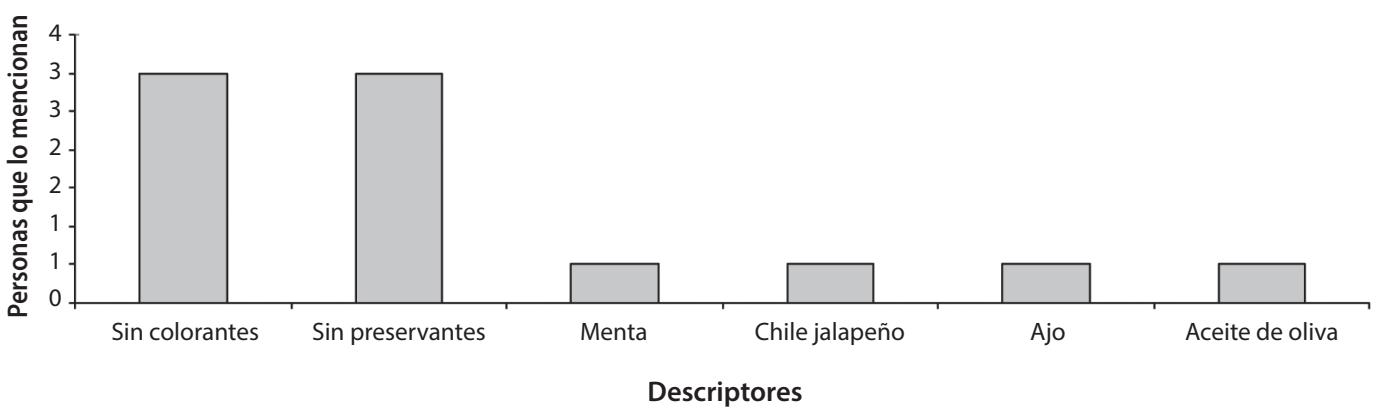

FIG. 5. Descriptores más representativos de los ingredientes del producto ideal generados por ambos grupos focales $(p=0,8095)$.

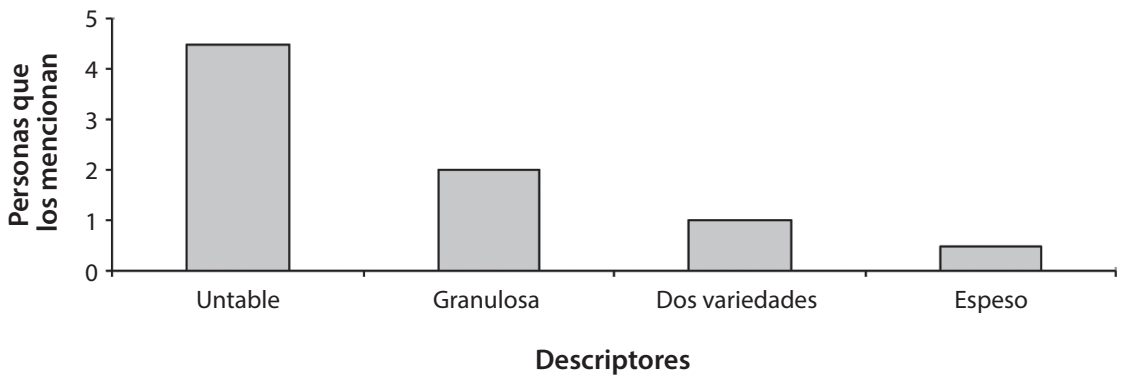

FIG. 6. Descriptores más representativos de la textura del producto ideal generados por ambos grupos focales $(p=0.729)$. 
Planetario: Las figuras 7 y 8 muestran los resultados obtenidos en esta sección.

Preferencia para acompañar el producto untable tipo hummus: El grupo 1 comentó que les gustaría acompañarlo con pan pita, chips y wrap de vegetales, mientras que el grupo 2 tuvo preferencias más variadas: galleta de soda, pan pita, sándwiches, huevo duro, vegetales, falafel, chips.

Tendencias en el desarrollo de productos: El grupo 1 mencionó los siguientes enfoques: saludable y exótico, seguridad alimentaria y variado. El grupo 2 mencionó lo siguiente: amigable con el ambiente, saludable, precio, natural, conveniencia, competir con lo exótico (por ser orgánico y natural) y variado.

Degustación: En general, los producto untable tipo hummus más gustados fueron el tradicional y jalapeño, sin embargo, las personas a las que les gustó el de perejil y culantro, les gustó mucho (Cuadro 1).

Evaluación de la competencia: La presentación en vidrio fue aceptada por ser elegante y ecológica, pero la presentación con tapa transparente y media etiqueta (como la competencia que existe en Costa Rica) fue la más aceptada de todas.

También se hizo énfasis en que si el producto es natural y saludable, su empaque debe ir acorde a este concepto, por lo que debe ser oxo biodegradable.

\section{DISCUSIÓN}

En este estudio se encontró una aceptación general del producto untable tipo hummus a base de la variedad criolla de gandul, para lo cual esta es una investigación pionera, aunque sí existen otros artículos que han estudiado el hummus a base de garbanzo (Al-Holy, Al-Qadiri, Lin \& Rasco, 2006; Ayed \& Emad, 2007; Friedman \& Juneja, 2010).

La curiosidad expresada por los participantes sobre el producto podría sugerir que sería aceptado por el consumidor después de un proceso de desarrollo de producto nuevo y estudio de mercado, aunque esta es sólo una hipótesis que debe probarse en la práctica.

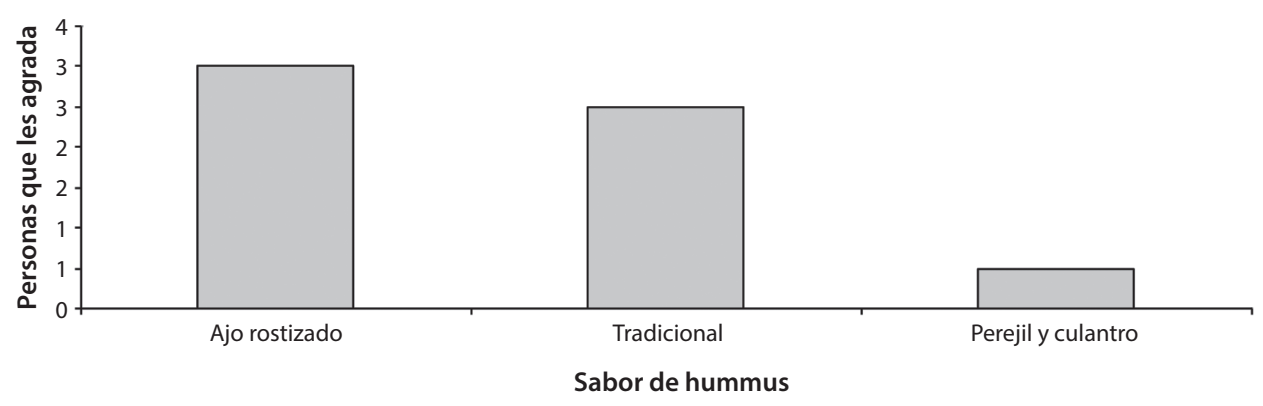

FIG. 7. Sabores de hummus que más gustaron a los dos grupos que realizaron los grupos focales $(p=0,9999)$.

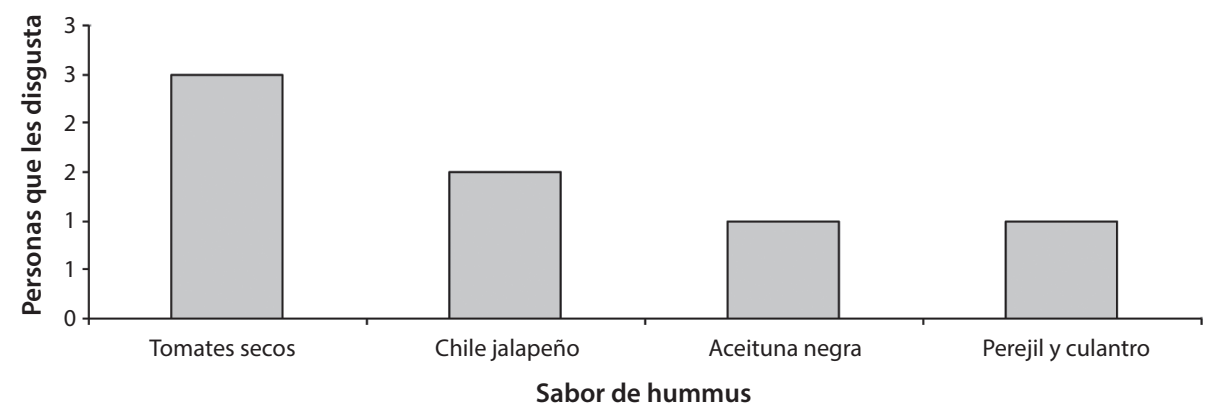

FIG. 8. Sabores de hummus que menos gustaron a los dos grupos que realizaron los grupos focales $(p=0,9784)$. 


\section{CUADRO 1}

Impresiones más importantes de la degustación realizada en ambos mini grupos focales sobre 4 producto untable tipo hummus a base de gandul

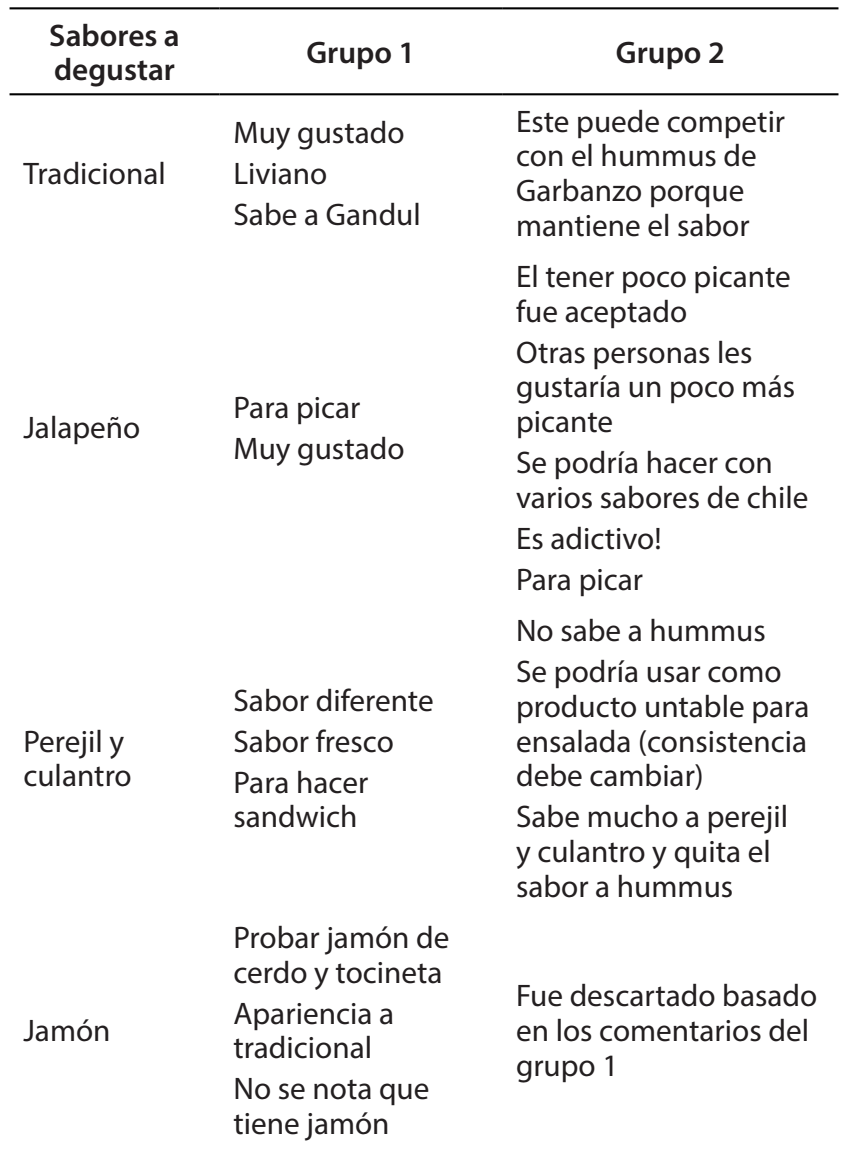

El color beige y natural preferidos por los participantes se equiparan con los que son vendidos actualmente por la competencia. Esto sugiere que esos son los colores con los que se deben seguir realizando las pruebas a nivel piloto de este producto.

El sabor a gandul sin sobresalir al resto de ingredientes como descriptor del producto es comparable con otro estudio en el que se desarrolló una salsa de tamarindo y uno de los descriptores determinantes para preferirla sobre la competencia es que la salsa supiera a tamarindo (Aguirre, 2009).

Las opiniones encontradas con respecto a la apariencia sin grumos y con grumos son semejantes a un estudio realizado por Aguirre (2009) en donde se aceptaba tanto la presencia o ausencia de trozos de vegetales o grumos en el producto de tamarindo que desarrolló.
La tapa transparente sugerida para el empaque y la información sobre el producto se asemejan con un estudio en el que se realizó un grupo focal para evaluar la calidad de carne de conejo y al evaluar el empaque del producto se menciona también que es conveniente utilizar un empaque en el que se pueda tanto ver el producto como colocar información del mismo (Chavez \& Chavez, 2006). Además, el que sea oxibiodegradable es positivo para el producto ya que es una tecnología que logra reducir la vida útil de los envases y evita la contaminación que producen los plásticos tradicionales en el ecosistema (IFA, 2012).

La ausencia de colorantes o preservantes es una característica demandada por los participantes así como una tendencia en la actualidad. Con respecto a esto, Restrepo (2007) estudió el uso de betacarotenos en los alimentos en vez de sustancias artificiales por sus propiedades antioxidantes y pigmentantes.

El haber encontrado diferentes razones por las cuales los consumidores preferirían este producto por encima del existente de garbanzo muestra su interés por el mismo y el potencial comercial que tendría en Costa Rica por ser un cultivo alternativo, práctica que se está desarrollando de diferentes formas tal y como Jaramillo, Castrillón y Gallego (2011), ya que han diseñado estrategias para impulsar el cultivo de yuca como sustituto de maíz. Además, Güemes (2007) estudió el uso de derivados de cereales y leguminosas como sustitutos de ingredientes en la elaboración de productos cárnicos.

Las contradicciones encontradas con respecto a la idea de consumir el producto con chile jalapeño sin degustarlo y después de degustarlo son similares a un estudio sobre mayonesa de Cardinal, Flores, Contarini y Hough (2003) y uno sobre una serie de batidos (smoothies) de Loma-Montoya, Naranjo-Chano y López-Villacís (2009), en dondea los integrantes no les agradaban las ideas de combinaciones pero cuando los probaban cambiaban de opinión y les gustaba.

Las tendencias de mercado definidas por los participantes para el producto son de vital relevancia para su desarrollo, ya que va dirigido a consumidores ya sea vegetarianos o selectos, que buscan variedad y si no se cumple con este requisito, el éxito del producto puede no ser el esperado. Según Blanco (2012) la innovación de productos alimenticios debe ir integrada con las tendencias del mercado en términos de inocuidad, medio ambiente, salud, información nutricional.

Se concluye que el producto untable tipo hummus agra$\mathrm{da}$ al costarricense pero es necesario un estudio de mercado que pueda generalizar lo anterior, ya que este estudio se realizó con una muestra pequeña de consumidores. 
Se concluye también que el producto untable ideal que se podría fabricar es color beige, untable, sin grumos, con empaque oxibiodegradable, de tapa plástica sin preservantes, colorantes y de sabor tradicional o chile jalapeño.

Se recomienda seguir investigando este producto ya que ha tenido aceptación entre las personas que lo han probado, especialmente utilizando la herramienta denominada QFD, en sus siglas en inglés (Quality Function Deployment) a partir de los resultados de este artículo y otras investigaciones que se realicen.

Además, se recomienda la investigación en los ámbitos de análisis físico químicos (para determinar los parámetros entre los que debe estar con respecto a la competencia), análisis microbiológicos (para determinar condiciones de planta) y análisis sensoriales para seguir afinando el proceso y las características del producto.

\section{AGRADECIMIENTOS}

Se agradece a todo el personal de la Escuela de Ciencias Exactas y Naturales por su esfuerzo y colaboración en la realización de los mini grupos focales que se elaboraron para este estudio.

\section{REFERENCIAS}

Al-Holy, M; Al-Qadiri, H; Lin, M., \& Rasco, B. (2006). Inhibition of Listeria innocua in Hummus by a combination of Nisin and Citric Acid. Journal of Food Protection, 69 (6), 13221327. Recuperado dehttp://www.ingentaconnect.com/ content/iafp/jfp/2006/00000069/00000006/art00012

Alinezad, A., Seif, A., \& Esfandiari, N. (2011). Supplier Evaluation and selection with QFD and FAHP in a pharmaceutical company.Int. Adv. Manuf Technology. Recuperado de http://link.springer.com/article/10.1007/ s00170-013-4733-3\#page-2

Aguirre M.V. (2009). Desarrollo de producto: Salsa de Tamarindo. (Tesis de grado para optar por título de Ingeniero en Alimentos). Universidad San Francisco de Quito, Ecuador. Recuperado dehttp://repositorio.usfq.edu.ec/bitstream/23000/1089/1/93016.pdf

Amerling, C. (2012). Receta de ensalada Gandul. Comunicación personal. Derechos reservados. San José, Costa Rica.

Aroca, D., Izaguirre, J., \& Zelaya, F. (2008). Identificación, demarcación y manejo de zonas de recarga hídrica en la Subcuenca Aguas Calientes. FOCUENAS II. Somoto, San Lucas. Recuperado de http://scholar.googleusercontent.com/ scholar?q=cache:UOcVz-6o0-oJ:scholar.google.com/+Ga ndul,+cuencas+hidrogr\%C3\%A1ficas\&hl=es\&as_sdt=0,5

Ayed, S.,\& Emad, I. (2007). Thermal processing requirements of canned chickpea dip. International Journal of Food Science and Technology,29(4), 441-448. Recuperado dehttp:// onlinelibrary.wiley.com/doi/10.1111/j.1365-2621.1994. tb02085.x/abstract

Barrios, E.X.,\& Costell, E. (2004). Review: Research into Consumers' Opinions and Attitudes in Food Research. Food Science and Technology International, 10, 359-371.

Blanco, A. (2012). Guía de estudio digital: Seminario de desarrollo de productos II. EUNED, San José, Costa Rica.

Bressani, R. Gómez, R.,\& Gonzaga, E. (1986). Calidad nutricional de la proteína del Gandul, tierno y maduro, y su valor suplementario a los cereales. Arch. Latinoam. Nutr., 36 (1),108-116.Recuperado de http://www.metabase.net/ docs/incap/07195.html

Boskou, D. (2012). MediTERRA: the Mediterranean diet for sustainable regional development: chapter 13: Presses de Sciences Po « Annuels ».CIHEAM. Recuperado dehttp://www.cairn. info/mediterra-2012-english--9782724612486.htm

Cardinal, P., Flores, A., Contarini, A.,\& Hough, G. (2003). Grupo focal research on sensory language used by consumers to describe mayonnaise. Journal of Sensory Studies, 18(1), 47-59. Recuperado de http://www.researchgate.net/publication/227820238_FOCUS_GROUP_RESEARCH_ON_ SENSORY_LANGUAGE_USED_BY_CONSUMERS_TO_DESCRIBE_MAYONNAISE

Chaves, A. (2010). Cocina vegetariana: recetas con garbanzo. Recetas by Suite101. Recuperado de http://suite101.net/ article/hummus-de-garbanzo-a21178

Chavez-Chavez, C.A.,\& Chavez-Flores V.E. (2006). Estrategia de desarrollo de marca para la carne de conejo de la granja Don Bosco.(Tesis para optar por el grado de licenciatura en mercadotecnia). Universidad Dr. José Matias Delgado, Facultad de Economía Dr. Santiago I. Barberena. Antiguo Cuscatlán, México. Recuperado dehttp://webquery.ujmd. edu.sv/siab/bvirtual/BIBLIOTECA\%20VIRTUAL/TESIS/01/ MER/ADCE0000302.pdf

Erraach, Y., Sayadi, S.,\& Parra-López, C. (2011). Quality function deployment (QFD) in the Spanish olive oil sector. Institute of Agricultural Research and training (IFAPA). Recuperado dehttp://ageconsearch.umn.edu/bitstream/114340/2/ Erraach_Yamna_351.pdf

Friedman, M.,\& Juneja, V. (2010). Review of antimicrobial and antioxidative activities of Chitosans in food. Journal of Food Protection, 73(9), 1737-1761. Recuperado de:http://www.ingentaconnect.com/content/iafp/ jfp/2010/00000073/00000009/art00021

Güemes, N. (2007). Utilización de los derivados de los cereales y leguminosas en la elaboración de productos cárnicos. NACAMEH, 1(2), 110-117. Recuperado de http://scholar. google.com/scholar?start=20\&q=Sustituto+de+la+harin a\&hl=es\&as_sdt $=0$

Hernández, J.,\& Izaguirre, J. (SF). Gandul: ficha técnica. CENADE. Recuperado dehttp://www.funica.org.ni/docs/product_ ani_17.pdf 
IFA. (2012). Envases oxo-biodegradables para los amenities de los hoteles Lopesan. Recuperado de https://www.lopesan. com/es/envases-oxo-biodegradables-para-los-amenities-de-los-hoteles-lopesan-20120604_007f88.html

Jaramillo, M., Castrillón, A.,\& Gallego, J. (2011). Diseño de estrategias para impulsar el cultivo masivo y tecnificado de la yuca en Colombia como producto sustituto del maíz en la elaboración de alimentos balanceados para la industria pecuaria. Especialización en Gerencia Agropecuaria. Recuperado de http://repository.lasallista.edu.co/dspace/ handle/10567/622

Khan, S., Saeed, Z., Hameed, H., Hussain, I., Amer, S.,\& Haq, M. (2011). Nutritional guidelines for school lunch programs: creating a culture of healthy eating at U.S. Islamic Schools. Public Health: IMANA. Recuperado dehttp://c.ymcdn. com/sites/www.imana.org/resource/resmgr/Files/Nutritional_Guidelines_for_S.pdf

Liberterre. (SF). Gandul: clasificación botánica. Recuperado de http://www.liberterre.fr/gaiagnostic/semillas/gandul.html

Loma-Montoya P.F., Naranjo-Chano, A.I.,\& López-Villacís, A. (2009). Elaboración y comercialización de nutrismoothie en la ciudad de Guayaquil. Proyecto de graduación previo a la obtención del título de: economista con mención en gestión empresarial, especialización marketing economista con mención en gestión empresarial. Facultad de
Economía y Negocios. Recuperado dehttp://www.dspace.espol.edu.ec/bitstream/123456789/10301/1/Proyecto\%20NutriSmoothie.pdf

Mena, C. (2012). Sobre nombre propio de productos comerciales. Comunicación personal. Registro de propiedad Industrial. San José, Costa Rica.

Ocampo, M. (2010). Generalidades sobre el Gandul. Comunicación personal. UNED, San José, Costa Rica.

Restrepo, M. (2007). Sustitución de colorantes en alimentos. Revista Lasallista de Investigación, 4(1), 35-39

Saravia, K. (2012). Receta guiso Gandul. Comunicación Personal. Derechos reservados. San José, Costa Rica.

Sharma, P. (1993). Conocimientos tradicionales agrosilvopastoriles y su adaptación a la conservación de los recursos naturales en la región Chorotega de Costa Rica. CATIE. Recuperado de http://books.google.co.cr/books?id=I_sOAQAAIAAJ\& $\mathrm{pg}=\mathrm{PA} 20 \& \mathrm{lpg}=\mathrm{PA} 20 \& \mathrm{dq}=\mathrm{Gandul},+$ fijador + nitr\%C3\%B3 geno\&source=bl\&ots=bu_xFEmcPw\&sig=PRaEqRBxuXX OlcY8Xjc6DtxFxC4\&hl=es\&sa=X\&ei=ur5mUJXVD4G09gS A2oGACQ\&ved=0CC0Q6AEwAw\#v=onepage \&q=Gandu I\%2C\%20fijador\%20nitr\%C3\%B3geno\&f=false

Stanton, W. (1990). Fundamentos del Marketing. México: McGraw Hill. 


\section{APÉNDICE 1}

\section{CUADRO AP1}

Descriptores del producto untable tipo hummus a base de gandul generados por el primer mini grupo focal integrado por 6 personas

\begin{tabular}{|c|c|c|c|c|c|c|}
\hline Participante & Color & Sabor & Apariencia & Empaque & Ingredientes & Textura \\
\hline 1 & $\begin{array}{l}\text { Color café } \\
\text { claro }\end{array}$ & $\begin{array}{l}\text { Característico } \\
\text { de Gandul } \\
\text { Picante }\end{array}$ & $\begin{array}{l}\text { Con pequeñas } \\
\text { partículas } \\
\text { sensibles al } \\
\text { paladar }\end{array}$ & $\begin{array}{l}\text { Tamaño: } \\
\text { 200g, Tapa } \\
\text { transparente } \\
\text { Con dibujos } \\
\text { referentes al } \\
\text { limón y al ajo }\end{array}$ & $\begin{array}{l}\text { Sin colorantes } \\
\text { Que se sienta } \\
\text { el ácido del } \\
\text { limón y el } \\
\text { ajo, la sal y } \\
\text { la pimienta, } \\
\text { paprika, hojas } \\
\text { de menta }\end{array}$ & $\begin{array}{l}\text { Semipastoso } \\
\text { Untable }\end{array}$ \\
\hline 2 & Pálido & $\begin{array}{l}\text { Que se sientan } \\
\text { las especies. } \\
\text { No muy salado. } \\
\text { Que se sienta } \\
\text { principalmente } \\
\text { el ajo. }\end{array}$ & Sin grumos & $\begin{array}{l}\text { Biodegradable, } \\
\text { Tipo cajas de } \\
\text { natilla }\end{array}$ & Chile jalapeño & $\begin{array}{l}\text { No muy seca ni } \\
\text { muy acuosa. } \\
\text { Que sirva para } \\
\text { untar }\end{array}$ \\
\hline 3 & Natural & $\begin{array}{l}\text { Sabor a } \\
\text { Gandul, } \\
\text { ajo, cebolla, } \\
\text { pimienta, sal, } \\
\text { culantro y } \\
\text { limón }\end{array}$ & $\begin{array}{l}\text { Que se sientan } \\
\text { los grumos. } \\
\text { Que se noten } \\
\text { los granitos de } \\
\text { Gandul }\end{array}$ & $\begin{array}{l}\text { En bolsa plástica } \\
\text { transparente } \\
\text { (tipo doypack } \\
\text { plástico) }\end{array}$ & $\begin{array}{l}\text { Sin colorantes, } \\
\text { pero con un } \\
\text { aditivo que } \\
\text { aumente su } \\
\text { vida útil }\end{array}$ & $\begin{array}{l}\text { Suave, pero lo } \\
\text { suficientemente } \\
\text { espeso para que } \\
\text { se pueda untar en } \\
\text { una tortilla }\end{array}$ \\
\hline 4 & Café & $\begin{array}{l}\text { Variedad de } \\
\text { sabores }\end{array}$ & $\begin{array}{l}\text { Textura } \\
\text { uniforme }\end{array}$ & $\begin{array}{l}\text { De plástico } \\
\text { Pequeño } \\
\text { Transparentes }\end{array}$ & Sin aditivos & Espeso \\
\hline 5 & $\begin{array}{l}\text { Amarillo } \\
\text { oscuro }\end{array}$ & $\begin{array}{l}\text { Sabor fuerte. } \\
\text { Que se sientan } \\
\text { las especies } \\
\text { Picante }\end{array}$ & $\begin{array}{l}30 \% \text { de } \\
\text { grumos }\end{array}$ & $\begin{array}{l}\text { Cartón } \\
\text { parafinado } \\
\text { Reciclable }\end{array}$ & $\begin{array}{l}\text { Colorante } \\
\text { natural (puede } \\
\text { ser chile) } \\
\text { Mucho ajo y } \\
\text { chile picante }\end{array}$ & $\begin{array}{l}\text { Espeso pero fácil } \\
\text { de untar }\end{array}$ \\
\hline 6 & $\begin{array}{l}\text { Lo más } \\
\text { blanco } \\
\text { posible }\end{array}$ & Picante & $\begin{array}{l}\text { Textura } \\
\text { uniforme }\end{array}$ & $\begin{array}{l}\text { Color blanco y } \\
\text { verde } \\
\text { Biodegradable }\end{array}$ & $\begin{array}{l}\text { Sin colorantes, } \\
\text { cebolla, ajo, } \\
\text { culantro, } \\
\text { Tocino y jamón }\end{array}$ & Untable \\
\hline
\end{tabular}


CUADRO AP2

Descriptores del producto untable tipo hummus a base de Gandul generados por el segundo mini grupo focal integrado por 6 personas.

\begin{tabular}{|c|c|c|c|c|c|c|}
\hline Participante & Color & Sabor & Apariencia & Empaque & Ingredientes & Textura \\
\hline 1 & $\begin{array}{l}\text { Pálido } \\
\text { blancuzco }\end{array}$ & $\begin{array}{l}\text { Que se le note } \\
\text { el limón, el ajo, } \\
\text { la sal pero que } \\
\text { no sea picante o } \\
\text { muy ácido }\end{array}$ & Con grumos & $\begin{array}{l}\text { Recipiente blanco } \\
\text { y azul, que refleje } \\
\text { que sea exótico. } \\
\text { Empaque podría } \\
\text { ser plástico con } \\
\text { dibujos que hagan } \\
\text { referencia al limón } \\
\text { y ajo }\end{array}$ & $\begin{array}{l}\text { Sin colorantes ni } \\
\text { preservantes }\end{array}$ & $\begin{array}{l}\text { Granulosa, suave } \\
\text { pero no demasiado o } \\
\text { esponjosa como un } \\
\text { lustre }\end{array}$ \\
\hline 2 & Caqui claro & $\begin{array}{l}\text { Que sepa } \\
\text { bastante a ajo y } \\
\text { aceite de oliva }\end{array}$ & $\begin{array}{l}\text { Delicada, } \\
\text { suave, sin } \\
\text { grumos }\end{array}$ & $\begin{array}{l}\text { Con tapa de plástico } \\
\text { transparente }\end{array}$ & $\begin{array}{l}\text { Sin colorantes o } \\
\text { preservantes. } \\
\text { Lo más natural } \\
\text { posible. }\end{array}$ & $\begin{array}{l}\text { Podría tener dos } \\
\text { variedades, según } \\
\text { para lo que se vaya } \\
\text { a acompañar. Que } \\
\text { no sea tan líquida, } \\
\text { pero que tenga una } \\
\text { consistencia que } \\
\text { permita untar, que no } \\
\text { sea tan seco o duro } \\
\text { que no permita untar. }\end{array}$ \\
\hline 3 & Claro & No muy salado & $\begin{array}{l}\text { Liso, sin } \\
\text { grumos } \\
\text { porque parece } \\
\text { que no está } \\
\text { bien hecho }\end{array}$ & $\begin{array}{l}\text { Llamativo con } \\
\text { decoraciones. } \\
\text { Transparente, que } \\
\text { se pueda ver el } \\
\text { color y textura. }\end{array}$ & $\begin{array}{l}\text { Sin colorantes } \\
\text { o preservantes, } \\
\text { ingredientes } \\
\text { quiero que tenga } \\
\text { aceite de oliva }\end{array}$ & $\begin{array}{l}\text { Espesa pero que se } \\
\text { pueda untar }\end{array}$ \\
\hline 4 & $\begin{array}{l}\text { Color beige } \\
\text { Es } \\
\text { importante } \\
\text { hacer las tres } \\
\text { variedades } \\
\text { de Gandul }\end{array}$ & $\begin{array}{l}\text { Con buen } \\
\text { aroma a } \\
\text { especias indias } \\
\text { o asiáticas, } \\
\text { porque le } \\
\text { da mucha } \\
\text { propiedad y } \\
\text { sabor. Además } \\
\text { del ajo, aceite } \\
\text { de oliva } \\
\text { (característico) y } \\
\text { ajonjolí. }\end{array}$ & Lisa y brillante & $\begin{array}{l}\text { Transparente que se } \\
\text { pueda observar. } \\
\text { Biodegradable. } \\
\text { Que en el } \\
\text { etiquetado describa } \\
\text { las propiedades del } \\
\text { producto, haciendo } \\
\text { énfasis en cada } \\
\text { variedad y poner los } \\
\text { beneficios de cada } \\
\text { una. }\end{array}$ & $\begin{array}{l}\text { Sin colorantes } \\
\text { o preservantes, } \\
\text { especies } \\
\text { orientales o curry }\end{array}$ & $\begin{array}{l}\text { Espumoso, fácil de } \\
\text { untar sobre pan pita, } \\
\text { vegetales o galletas, } \\
\text { que no sea muy seco, } \\
\text { es como un puré. }\end{array}$ \\
\hline 5 & Beige & $\begin{array}{l}\text { Ácido } \\
\text { Fresco. Picante } \\
\text { (paprika, chile } \\
\text { jalapeño o } \\
\text { chile mexicano } \\
\text { seco, o chile } \\
\text { limonense o } \\
\text { panameño) }\end{array}$ & $\begin{array}{l}\text { Homogéneo, } \\
\text { pero que se } \\
\text { noten los } \\
\text { ingredientes } \\
\text { que tiene }\end{array}$ & $\begin{array}{l}\text { Similar a cualquier } \\
\text { producto untable } \\
\text { tipo hummus }\end{array}$ & Tradicional & Fácil de untar \\
\hline 6 & $\begin{array}{l}\text { Parecido al } \\
\text { hummus de } \\
\text { garbanzo }\end{array}$ & $\begin{array}{l}\text { Un poco } \\
\text { picante }\end{array}$ & Sin grumos & $\begin{array}{l}\text { Color mejor que } \\
\text { sea acorde con el } \\
\text { producto }\end{array}$ & $\begin{array}{l}\text { Con perejil y } \\
\text { culantro }\end{array}$ & $\begin{array}{l}\text { Textura pastoso, sino } \\
\text { no es hummus }\end{array}$ \\
\hline
\end{tabular}


